\title{
Molecular and social regulation of worker division of labour in fire ants
}

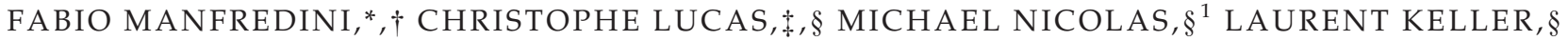 \\ DEWAYNE SHOEMAKER $\|$ and CHRISTINA M. GROZINGER* \\ *Department of Entomology, Center for Pollinator Research, The Pennsylvania State University, University Park, PA 16802, \\ USA, †School of Biological Sciences, Royal Holloway University of London, Egham, TW 20 0EX, UK, \$Institut de Recherche \\ sur la Biologie de l'Insecte (UMR 7261), CNRS, University of Tours, Parc de Grandmont, 37200 Tours, France, §Department of \\ Ecology \& Evolution, University of Lausanne, Biophore, Unil-Sorge, Lausanne, Switzerland, \\ ๆUSDA-ARS, Gainesville, FL 32608, USA
}

\begin{abstract}
Reproductive and worker division of labour (DOL) is a hallmark of social insect societies. Despite a long-standing interest in worker DOL, the molecular mechanisms regulating this process have only been investigated in detail in honey bees, and little is known about the regulatory mechanisms operating in other social insects. In the fire ant Solenopsis invicta, one of the most studied ant species, workers are permanently sterile and the tasks performed are modulated by the worker's internal state (age and size) and the outside environment (social environment), which potentially includes the effect of the queen presence through chemical communication via pheromones. However, the molecular mechanisms underpinning these processes are unknown. Using a whole-genome microarray platform, we characterized the molecular basis for worker DOL and we explored how a drastic change in the social environment (i.e. the sudden loss of the queen) affects global gene expression patterns of worker ants. We identified numerous genes differentially expressed between foraging and nonforaging workers in queenright colonies. With a few exceptions, these genes appear to be distinct from those involved in DOL in bees and wasps. Interestingly, after the queen was removed, foraging workers were no longer distinct from nonforaging workers at the transcriptomic level. Furthermore, few expression differences were detected between queenright and queenless workers when we did not consider the task performed. Thus, the social condition of the colony (queenless vs. queenright) appears to impact the molecular pathways underlying worker task performance, providing strong evidence for social regulation of DOL in $S$. invicta.
\end{abstract}

Keywords: division of labour, fire ants, foraging workers, microarrays, queen pheromone, sociogenomics

Received 7 October 2013; revision received 1 December 2013; accepted 6 December 2013

\section{Introduction}

Social groups often exhibit a division of labour (DOL), characterized by a differentiation in the tasks performed

Correspondence: Fabio Manfredini, Fax: +44 1784 414224; E-mail: fmanfredini79@gmail.com

${ }^{1}$ Present address: Department of Plants Molecular Biology, Centro Nacional de Biotecnologia, C/Darwin 3, Campus de Cantoblanco (UAM), 28049 Madrid, Spain by the group members, a process that is believed to enhance the overall efficiency of the group (Duarte et al. 2011). While DOL is found in social groups of many species, including birds (Arnold et al. 2005), meerkats (Manser 1999), dolphins (Gazda et al. 2005) and humans (Durkheim \& Coser 1997), the most sophisticated and best studied cases of DOL are found among highly eusocial insects, namely bees, ants, wasps and termites (reviewed in Smith et al. 2008). These insect societies exhibit a reproductive DOL, where a few individuals 
develop into the reproductive caste, while most of the colony members become nonreproductive workers and perform all tasks related to colony maintenance and growth (Robinson 1992). In addition, social insects display worker DOL, where different individuals specialize on particular colony tasks such as brood care, nest building, defence and foraging (Wilson 1971). Recent studies suggest that similar molecular pathways involved in core biological processes (i.e. reproduction, nutrition and metabolism) are responsible for DOL in different insect lineages with independent origins of eusociality, such as honey bees (Amdam et al. 2004), ants (Corona et al. 2013) and wasps (Toth et al. 2007). This concept, known as the 'groundplan' hypothesis (reviewed in Johnson \& Linksvayer 2010), postulates that these molecular pathways derive from physiological traits present in the solitary ancestors that were co-opted and selected to evolve into the queen and worker castes of eusocial insects. However, more studies are needed to test this hypothesis in other systems and to confirm its validity at a broader scale.

Many factors can influence DOL in insect societies, including morphology, genetic variation, developmental and nutritional factors and experience (reviewed in Duarte et al. 2011). Further, in many social insect species, pheromones play an important role in regulating both reproductive and worker DOL (Wyatt 2003). Pheromones may have a primer or releaser effect: primer pheromones affect long-term physiological or endocrine processes in the recipient followed by delayed changes in behavioural responses, whereas releaser pheromones elicit immediate behavioural responses (Vander Meer et al. 1998; Blomquist \& Bagnères 2010). The social insect in which pheromones have been characterized most extensively is the honey bee Apis mellifera (reviewed in Grozinger accepted; Slessor et al. 2005). The pheromones released by the honey bee queen and the developing larvae (brood) can act as primer pheromones, inhibiting worker ovary activation and worker behavioural maturation from nursing/brood care to foraging (Pankiw et al. 1998; Hoover et al. 2003; Le Conte et al. 2006). Both pheromones also may act as releaser pheromones, stimulating attraction in the case of queen pheromone and brood feeding or pollen foraging in the case of brood pheromone (Dreller et al. 1999; Keeling et al. 2003). Recent microarray studies have revealed that exposure to either queen pheromone or brood pheromone can alter the expression of thousands of genes in the brains of worker honey bees, and these genes are associated with behavioural maturation/ worker DOL (Grozinger et al. 2003; Alaux et al. 2009). Thus, these studies suggest that genomic approaches can be used to both identify primer pheromones and characterize their impacts on behaviour and physiology.
Little is known about the molecular and social mechanisms that regulate worker DOL in ant colonies (reviewed in Libbrecht et al. 2013; Lucas et al. 2010; Lucas \& Sokolowski 2009). One of the best studied ant species is the fire ant Solenopsis invicta. The broad knowledge about the basic biology of this insect (reviewed in Tschinkel 2006), together with the understanding of the genetic regulation of social organization (Wang et al. 2013) and the recent sequencing of the genome (Wurm et al. 2011), makes S. invicta an emerging model in the field of sociogenomics (reviewed in Robinson et al. 2005). Fire ants live in large colonies with either a single queen (monogyne form) or multiple queens (polygyne form), and tens of thousands of workers organized in several behavioural phenotypes that are associated with size and age (Mirenda \& Vinson 1981). Queens produce a pheromone that seems to regulate many of the same behavioural and physiological processes as queen pheromone in honey bees, eliciting both primer and releaser responses. As a primer pheromone, it prevents virgin queens from shedding wings and activating ovaries (Fletcher \& Blum 1981; Vargo 1998), it affects caste determination of female larvae (Vargo \& Fletcher 1986a,b, 1987), and it may alter aggression levels of workers towards nestmates and acceptance of newly mated queens (Klobuchar \& Deslippe 2002; Vander Meer \& Alonso 2002). As a releaser, it elicits worker attraction to the queen and induces workers to groom and feed the queen (Vander Meer et al. 1980). Finally, in polygyne colonies, the individual egg-laying rate decreases as the number of queens increases owing to a primer pheromone produced by the various queens (Vargo 1992). The active chemical components of queen pheromone have not been characterized, but queens in different reproductive states differ in their venom alkaloid and hydrocarbon profiles (Eliyahu et al. 2011). However, the impacts of fire ant queen pheromone on worker DOL and the molecular mechanisms by which queen pheromone alters worker behaviour and physiology are unknown. In particular, given that fire ant workers are permanently sterile, the mode of action of the queen primer pheromone might be significantly different from the honey bee system where workers can activate their ovaries in the absence of a functional queen (Hoover et al. 2003).

In this study, we used whole-genome fire ant microarrays (Manfredini et al. 2013) to examine (i) the molecular basis for DOL in fire ant workers by comparing foraging and nonforaging workers and (ii) the impact of queen presence on worker gene expression patterns in these two behavioural groups. We hypothesized that similar genes/gene pathways that have been described in other genomic studies to be major regulators of worker DOL, specifically in-nest behaviours vs. foraging, in 
social insects such as honey bees and wasps (Alaux et al. 2009; Toth et al. 2010; Ament et al. 2011) would be involved in fire ant worker DOL. We also hypothesized that primer effects of queen pheromone would be evident at the gene expression level and that these effects would differ between the two behavioural groups.

\section{Materials and methods}

\section{Insect collection, rearing and sampling}

Monogyne colonies of Solenopsis invicta were collected near Athens (GA, USA) in April 2008 for Experiment 1 and in the surroundings of Gainesville (FL, USA) in April-May 2010 for Experiment 2. Fire ant colonies were kept under standard laboratory conditions (Jouvenaz et al. 1977) for 3 months before sampling. For Experiment 1, we sampled two groups of ants, that is, foraging (out) and nonforaging (in) workers, while for Experiment 2 the treatment groups were 4: queenright nonforaging (QRin), queenless nonforaging (QLin), queenright foraging (QRout) and queenless foraging (QLout). See Fig. S1 and Appendix S1 (Supporting Information) for a detailed description of rearing condition, group assignment and sampling methodology.

\section{Sample preparation for molecular analyses}

Total RNA was extracted from pools of 10 worker ants (whole bodies) using the PicoPure RNA Isolation kit (Applied Biosystems - Life Technologies, Grand Island, NY, USA) combined with an RNase-Free DNase step (Qiagen, Valencia, CA, USA) to remove any possible contamination by genomic DNA. Subsequent steps in the microarray analysis were performed at the Penn State Genomic Core Facility as in Manfredini et al. (2013) and see Appendix S1 (Supporting Information).

\section{Microarray analysis}

Our microarray, recently developed and validated (Manfredini et al. 2013), includes 51531 probes that match unique transcripts obtained from the sequencing of the S. invicta genome (Wurm et al. 2011) plus additional sequences from transcriptome studies. Probes were printed in pairs on two 12-plex microarrays (each array had a 135000 probe capacity, Roche NimbleGen, Inc., Madison WI, USA). We used a loop design with dye swaps incorporated. For Experiment 1, we used 6 arrays of a 12-plex microarray slide which allowed us to hybridize 12 RNA samples: these corresponded to 6 pools of foraging and 6 pools of nonforaging workers from six different colonies (Fig. S2, Supporting Information). For Experiment 2, we used all 12 arrays of a 12-plex microarray slide to hybridize 24 RNA samples: again, we used six different colonies, and for each colony, we processed 1 pool of QRin, 1 of QLin, 1 of QRout and 1 of QLout (Fig. S3, Supporting Information). Array data were analysed using two statistical software packages, namely R 2.11.1 (Team 2009) and SAS (Cary, NC, USA), for both experiments (Appendix S1, Supporting Information).

\section{Gene ontology and comparative analyses}

Hierarchical clustering using the Ward method and principal component analysis (PCA) for global patterns of gene expression were performed in JMP 9.0.2 (SAS, Cary, NC, USA), while K-means clustering was performed in GENESIS (Sturn et al. 2002). Gene ontology (GO) analyses were performed using functional annotation clustering in DAVID version 6 (Huang et al. $2009 a, b)$ with medium stringency and a cut-off of $P<0.05$. Fire ant genes were matched to their putative Drosophila orthologs in FlyBase (Marygold et al. 2013). To identify the most overrepresented biological functions (enrichment analysis), we compared the annotation composition in our list of differentially expressed genes to that of a population background composed by all the fire ant transcripts with Drosophila orthologs that were included in the statistical analysis. For comparative studies, we used Venny (Oliveros 2007) to overlap lists of differentially expressed genes (only those provided with FlyBase numbers) and we used a hypergeometric test (http:// nemates.org/MA/progs/overlap_stats.html) to assess whether genes overlapping between studies occurred significantly more often than expected by chance. Lists of significantly enriched GO terms obtained with functional annotation chart in DAVID (medium stringency and $P<0.05$ ) from different experiments were also overlapped in Venny.

Validation of differential expression of candidate genes using quantitative real-time PCR

We examined gene expression levels of seven candidate genes that were differentially expressed in one or both of the two microarray experiments and are known for regulating interesting biological functions in model organisms: foraging (for, food-related behaviour and polyethism), hymenoptaecin (Hym, antibacterial response), myofilin ( $m f$, muscle development), myosin heavy chain (mhc, locomotion), ornithine aminotransferase precursor (oat, neurogenesis), serine protease immune response integrator (spirit, innate immune response) and synaptotagmin 1 (syt1, neurotransmitter secretion). Expression levels were assayed in QRin, QRout and QLin groups by means of 
quantitative real-time PCR as in Manfredini et al. (2013), see Appendix S1 (Supporting Information).

\section{Results}

Experiment 1: gene expression patterns associated with task performed

The number of differentially expressed transcripts in Experiment 1 between foraging and nonforaging workers ('out' and 'in', respectively) was 1387 at False Discovery Rate (FDR) $<0.05$ (Table S1, Supporting Information), representing only $2.7 \%$ of the 51531 transcripts included in the analysis. The global analysis of patterns of expression revealed that the major driver was the task performed by workers. A hierarchical clustering analysis of the 12 individual pools of workers grouped them into two distinct macroclusters, the first encompassing 6 pools of foraging ants and the second 6 pools of nonforaging ants (Fig. 1A). A principal component analysis on the same data set confirmed this result, with the first component (which was associated with the task performed by the workers) accounting for $57.2 \%$ of the difference in gene expression. Overall, foraging and nonforaging ants clustered separately into two different portions of the space (Fig. 1B).

We performed gene ontology analysis on the differentially expressed transcripts that have Drosophila orthologs with FlyBase annotations (735 of 1387) using DAVID (Huang et al. 2009a,b). Six GO terms and 3 KEGG pathways were significantly enriched at $P<0.05$ (functional annotation clustering, Table S2, Supporting Information): generation of precursor metabolites and energy, myofibril assembly, muscle cell differentiation, multiorganism process, response to oxidative stress, monocarboxylic acid metabolic process, oxidative phosphorylation, pentose and glucuronate interconversions and lysine degradation.

\section{Experiment 2: effect of the presence of the queen on gene expression patterns}

We compared gene expression levels between four groups of ants for this experiment: foraging and nonforaging workers from queenright (QRout and QRin, respectively) and queenless colony fragments (QLout and QLin, respectively). Four-hundred transcripts were significantly differentially expressed across the four groups (FDR $<0.05$ ). Because the analysis with SAS in this case detected very few transcripts differentially expressed across treatments (see Appendix S1, Supporting Information), we performed a global analysis of patterns of expression on the whole set of transcripts that were initially included in the analysis. A hierarchi- cal clustering analysis revealed that, similarly to Experiment 1 , the task performed was the major driver of gene expression: nonforaging ants (QRin and QLin) formed a separate cluster from foraging workers (QRout and QLout), independent of the presence or absence of the queen (Fig. 2A). This result was confirmed by a principal component analysis, in which the first component corresponded to the task performed (foraging vs. nonforaging) and explained 54\% of the differences in gene expression, whereas social environment (presence or absence of the queen, second component) accounted for $25 \%$ of the differences. A third component, explaining $21 \%$ of the variation, revealed an interaction between task and social environment (Fig. 2B).

Pairwise comparisons of the four groups identified 395 transcripts that were differentially expressed between QRin and QRout at FDR < 0.05 (Table S3, Supporting Information), while there were very few transcripts differentially expressed in the other contrasts at this FDR (Table 1). We performed gene ontology analysis on the set of 395 transcripts significantly differentially expressed between QRin and QRout workers: of these, 248 have Drosophila orthologs with FlyBase annotations and were included in the functional annotation clustering in DAVID. We found that 7 GO terms and 2 KEGG pathways were significantly enriched (Table S4, Supporting Information). Among significant GO terms, ageing and cellular respiration survived the Benjamini correction (Benjamini and Hochberg 1995) at $P<0.05$; the others were skeletal myofibril assembly, aerobic respiration, organic acid metabolic process, signal transduction and larval central nervous system remodelling. Significant KEGG pathways were citrate cycle (TCA cycle), which survived the Benjamini correction at $P<0.05$, and starch and sucrose metabolism.

Because our analyses did not reveal a significant difference between queenright and queenless workers, we performed a second analysis where we pooled the transcripts that were differentially expressed either in the QRin/QLin or in the QRout/QLout comparisons and used a less stringent FDR $(<0.1)$. This analysis revealed 27 transcripts, of which 22 had Drosophila orthologs with FlyBase annotations (Table S5, Supporting Information). Among these genes were oat, hym, syt1, aspartyl beta-hydroxylase (Asph), cuticular protein 49Aa, NiemannPick type C-2 (Npc2a) and twin of eyeless (toy).

\section{Comparisons of significantly regulated transcripts in Experiments 1 and 2}

Interestingly, the only contrast that produced differentially expressed transcripts at FDR $<0.05$ in Experiment 2 was the comparison between foraging and nonforaging workers in queenright colonies, which is analogous to 

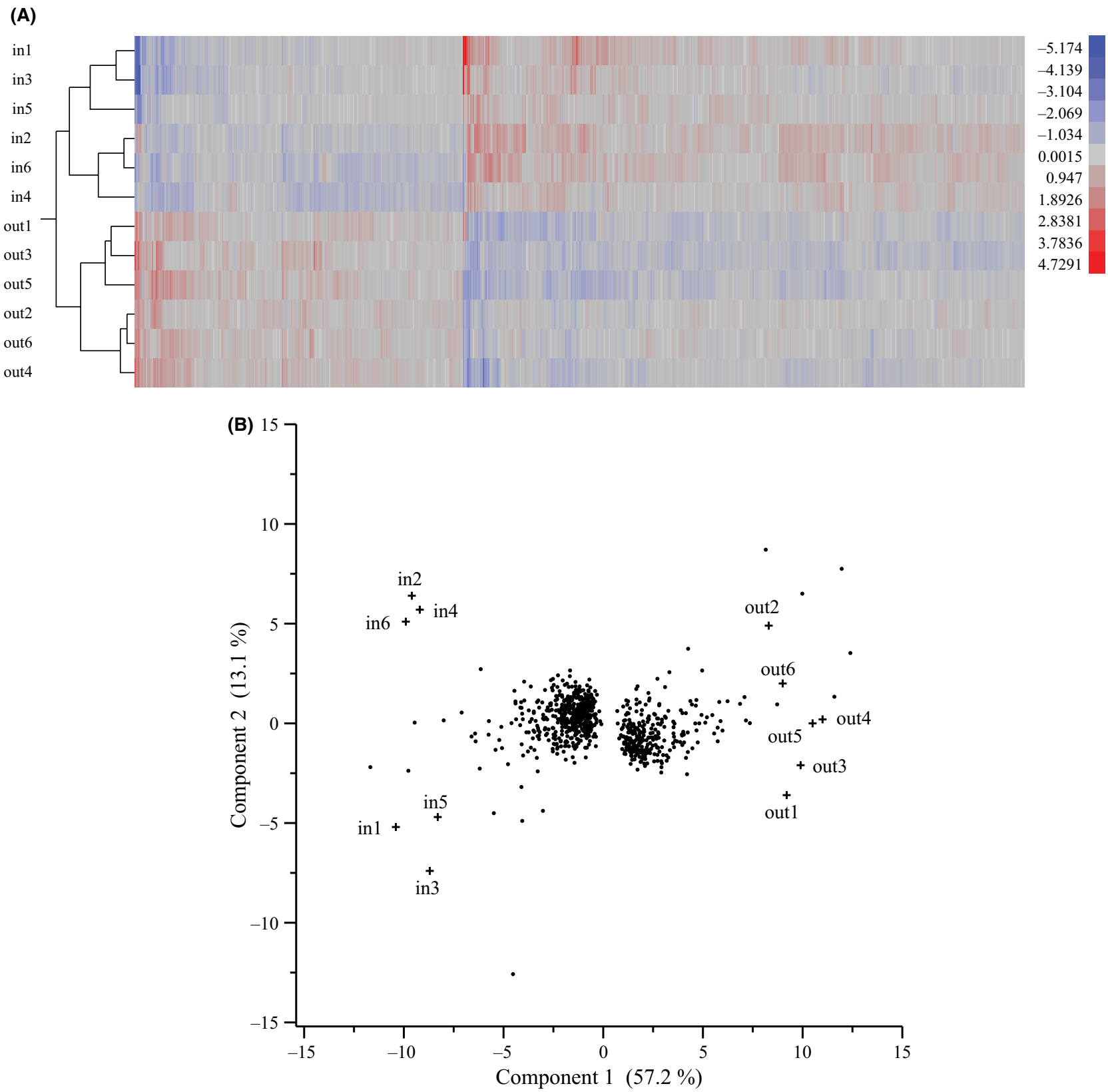

Fig. 1 Molecular regulation of worker division of labour. Global analyses of significantly differentially expressed transcripts in pools of workers from individual colonies (Experiment 1) show that patterns of gene expression were shared among nonforaging (in) and foraging (out) workers. (A) Heatmap of $\log _{2}$-transformed and normalized expression values and hierarchical clustering of worker pools according to expression patterns; (B) principal component analysis.

what we tested in Experiment 1. This allowed us to perform an overlap of transcripts that were differentially expressed in Experiments 1 and 2 (Fig. S5, Supporting Information). The overlap analysis identified 89 transcripts that were shared between the two experiments (Table S6, Supporting Information), a number significantly higher than expected by chance (hypergeometric test: representation factor: $\left.8.4, P<7.16 \mathrm{e}^{-55}\right)$. This group includes several genes that are well characterized in Drosophila, such as $m f, m h c$, oat, spirit, syt1, adipokinetic hormone receptor (Akhr), I'm not dead yet (Indy), cuticular protein 47Ef, cytochrome P450 (Cyp4 g1 and Cyp4c3), centrosomin (cnn), supercoiling factor (scf) and TBP-associated factor 8 (Taf8). A gene ontology analysis on the 63 transcripts with Drosophila orthologs and FlyBase annotations revealed that none of the 11 clusters produced by 
(A)
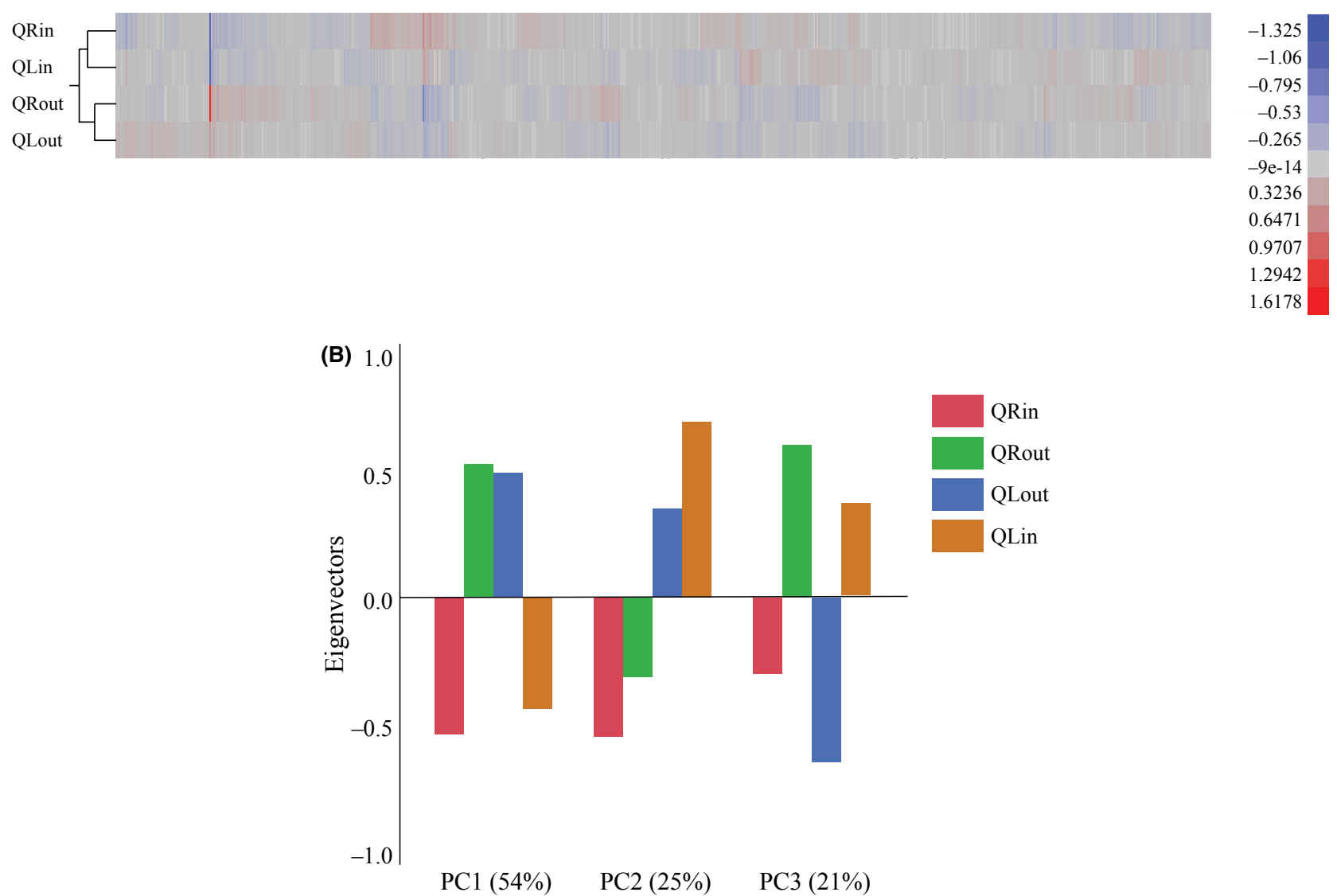

Fig. 2 Effect of social environment on the regulation of worker division of labour. Global analyses of the complete set of transcripts from Experiment 2 (foraging and nonforaging workers from queenright or queenless colonies). Hierarchical clustering (A) and principal component (B) analyses reveal that patterns of gene expression were more similar in nonforaging workers ('in' groups) and foraging workers ('out' groups) independently on the presence/absence of the queen. QRin, queenright nonforaging; QRout, queenright foraging; QLin, queenless nonforaging; QLout, queenless foraging.

the functional annotation clustering in DAVID was significantly enriched. We performed another overlap analysis on this same data set, this time using significantly enriched GO terms from each experiment instead of individual genes. Twelve GO terms were shared between the two experiments (Table S7, Supporting Information).

The lack of any significant difference in gene expression between foraging and nonforaging workers in queenless colonies from Experiment 2 suggests that in the absence of the queen, there is a breakdown in the performance of defined tasks (foraging vs. nonforaging) in workers. In honey bees, loss of the queen results in accelerated behavioural maturation, and workers enter the foraging state faster (Pankiw \& Page 2001). We investigated whether the gene expression patterns in queenless workers from Experiment 2 were more similar to foraging or nonforaging ants from Experiment 1 by using a directional overlap analysis of lists of transcripts obtained with the sAS protocol. We overlapped the complete set of 17960 transcripts that were upregulated (but not necessarily statistically significant) in queenless workers in Experiment 2 with significantly upregulated transcripts in nonforaging and foraging workers from Experiment 1 (Table S8, Supporting Information). For the queenless/foraging workers comparison, 292 transcripts were upregulated in both groups, while 351 were not (total of 643 transcripts); for the queenless/nonforaging workers comparison, 243 transcripts were upregulated in both groups, while 470 were not (total of 713 transcripts). This difference was highly significant (chi-square, $P<0.0001$ ), indicating that queenless workers were more 'forager-like' in terms of gene expression.

\section{Comparative studies across species}

To investigate whether mechanisms of division of labour are conserved across social insects, we performed 
Table 1 Pairwise comparisons of transcripts that were differentially expressed in the four groups of workers at two different False Discovery Rate (FDR) after analysis with $\mathrm{R}$

\begin{tabular}{cccccc}
\hline & \multicolumn{2}{l}{ FDR $<0.05$} & & \multicolumn{2}{l}{ FDR $<0.1$} \\
\cline { 2 - 3 } \cline { 5 - 6 } & $\mathrm{Up}$ & Down & & $\mathrm{Up}$ & Down \\
\hline $\begin{array}{c}\text { Nonforaging } \\
\text { QL vs. QR }\end{array}$ & 0 & 0 & & $14^{*}$ & $1^{*}$ \\
$\begin{array}{c}\text { Foraging } \\
\text { QL vs. QR }\end{array}$ & 0 & 4 & & $7^{*}$ & $5^{*}$ \\
$\begin{array}{c}\text { Queenright } \\
\text { In vs. out } \\
\begin{array}{c}\text { Queenless } \\
\text { In vs. out }\end{array}\end{array}$ & 89 & 306 & & 256 & 681 \\
\hline
\end{tabular}

$\mathrm{Up}=$ transcripts upregulated in the first term of the comparison; Down = transcripts downregulated in the first term of the comparison; * for these comparisons, the LIMMA method 'nested' was used instead of 'separate' to control for multiple testing across comparison because it was more powerful in detecting significantly regulated transcripts.

overlap analyses between all significantly differentially expressed genes from Experiment 1 (foraging vs. nonforaging ants in queenright colonies, 735 transcripts with FlyBase numbers) and the differentially expressed genes from previous studies on honey bees (Alaux et al. 2009; Ament et al. 2011) and paper wasps (Toth et al. 2010) that also investigated transcriptomic differences between foraging vs. nonforaging workers. Statistical analysis revealed that in both cases there was less overlap than expected by chance (see Appendix S1 and Table S9, Supporting Information). Only 16 genes were shared between S. invicta, A. mellifera and Polistes metricus, including $m f$, thiolester-containing protein II (Tep2), Rab-protein 7 (Rab7), probable cytochrome P450 6 g2, larval serum protein 2 (Lsp2), histone (His3.3A and His3.3B) and epidermal stripes and patches (Esp) (Fig. 3). We can add to this list for and syt1, both of which were differentially expressed in our study and in wasp (Toth et al. 2010) and in other honey bee studies (Ben-Shahar et al. 2002; Whitfield et al. 2003). The patterns of expression of these 16 genes in foraging and nonforaging workers were not consistent across the three social insects (data not shown).

\section{Discussion}

We investigated the molecular and social mechanisms underpinning worker division of labour (DOL) in the fire ant Solenopsis invicta. Our first major finding confirms that worker DOL in fire ants is associated with important changes at the transcriptomic level: 1387 transcripts in Experiment 1 and 395 transcripts in Experi- ment 2 were differentially expressed between foraging and nonforaging workers, and there was a significant overlap in the suites of differentially expressed transcripts between the two experiments. Furthermore, pools of ants from individual colonies clearly clustered into one of two groups based on expression profiles in Experiment 1. It is worth noting that despite the significant overlap, there were clear differences in the suites of genes differentially regulated between foraging and nonforaging workers in the two experiments. These differences are likely due to extrinsic factors related to the experimental conditions (e.g. year and site of collection in the field, colony genetic backgrounds, nest design, time of sampling) which were similar but not identical in the two experiments (see 'Insect collection, rearing and sampling' in the Appendix S1, Supporting Information).

Interestingly, the transcriptional differences between foraging and nonforaging workers completely disappeared in queenless conditions (Experiment 2), where only one gene (hypothetical protein SINV_01841) was differentially expressed between foraging and nonforaging workers. Despite the fact that social environment (presence/absence of the queen) contributed to $25 \%$ of the variation in gene regulation at a genomic scale, there were only a handful of genes significantly differentially expressed between queenless and queenright workers. Overall, these results suggest that DOL is transcriptionally regulated in fire ants and is associated with easily distinguishable behavioural (foraging/nonforaging) and spatial (inside/outside the nest) phenotypes. Furthermore, the queen does have primer effects on workers and appears to impact DOL, because because a queen's results in a more 'forager-like' gene expression patterns in workers.

Finally, comparative studies across species revealed a large variability in the proportion of transcripts differentially expressed according to foraging/nonforaging behaviour: these were $2.7 \%$ in fire ant whole bodies (Experiment 1), $4 \%$ in paper wasp brains, $13 \%$ in honey bee brains and $20 \%$ in honey bee fat bodies. Comparative studies also suggest that there is no substantial conserved suite of 'DOL genes' across these three species: overlap analyses were not supported by statistical significance and the directionality of the expression patterns of the 16 common genes varied from species to species. However, our results show some conservation of gene ontology categories when comparing fire ants with honey bees, while no GO terms were shared with paper wasps, possibly because for paper wasps only brain tissue was used. An alternative explanation for the negative results of our comparative analyses across social insects could be associated with the methodology of analysis itself. In fact, in order to directly compare gene regulation in different species of insects, we rely 


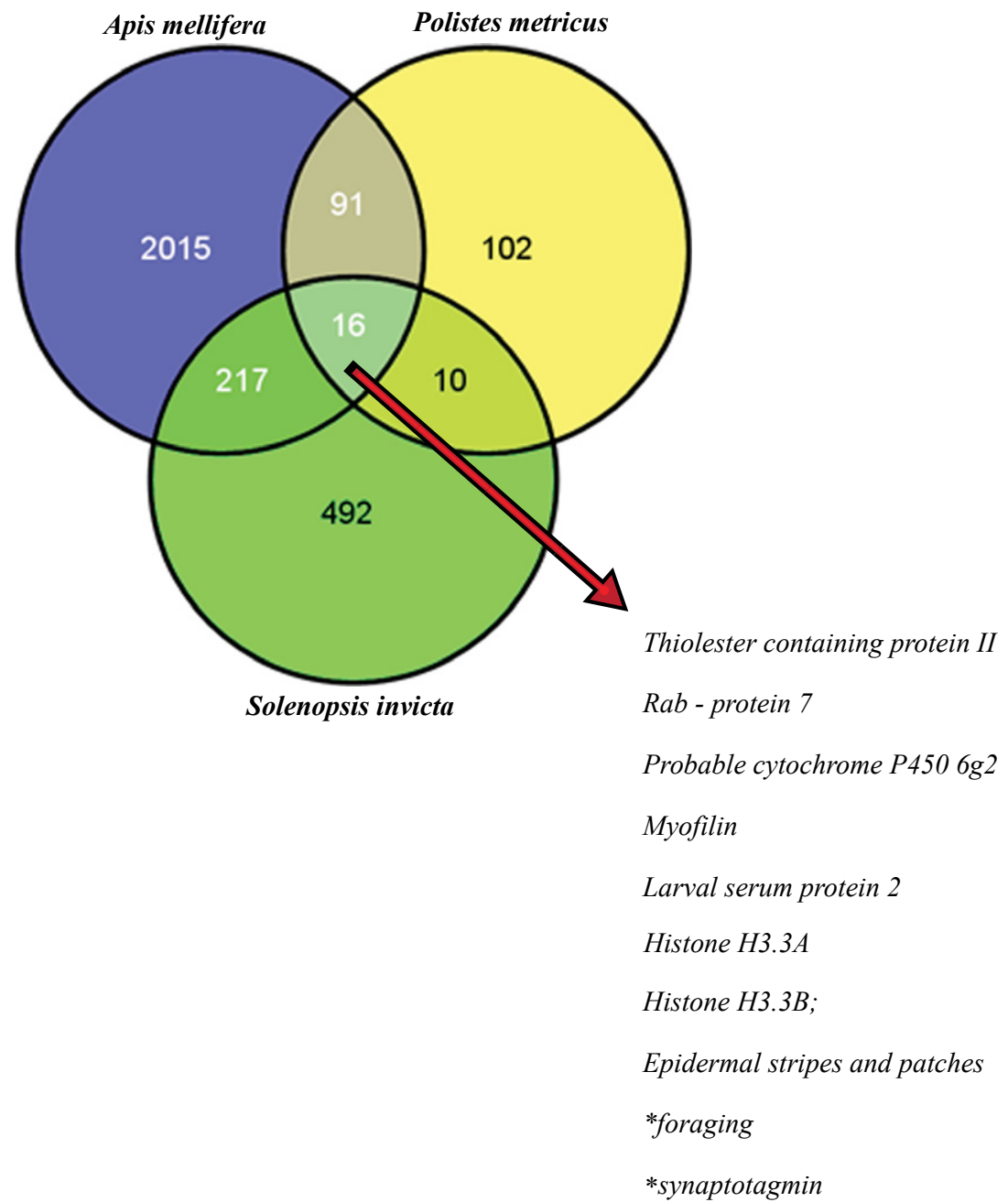

Fig. 3 Comparative analysis of transcripts associated with worker division of labour across social insect species. Overlap analysis of FlyBase numbers corresponding to transcripts that were differentially expressed between foraging and nonforaging workers in Apis mellifera (brain tissue, Alaux et al. 2009 and fat bodies Ament et al. 2011), Polistes metricus (brain tissue, Toth et al. 2010) and S. invicta whole-body samples from Experiment 1. *These genes were not listed in Alaux et al. 2009 and Ament et al. 2011, but were differentially expressed in A. mellifera based on different studies (Ben-Shahar et al. 2002; Whitfield et al. 2003). on annotations from Drosophila melanogaster, and while doing this, we might miss groups of genes that have specific functions in social insects or gene families that are expanded in this group but have not been characterized in D. melanogaster. Finally, comparisons between more closely related species (other ants) would likely reveal greater conservation of DOL genes.

\section{Molecular regulation of worker division of labour}

Gene ontology analyses in foraging and nonforaging workers revealed that muscle structure development and metabolic process recurred as differentially regulated across both experiments. Muscle activity is likely associated with differential motility and locomotory behaviour in the two groups of ants, where foraging workers actively search for food sources in the foraging area, while nonforaging workers perform nursing tasks within the nest. Muscle development also depends on the size and the age of an ant and these two features are good predictors for foraging and nonforaging behaviours in fire ant workers (Mirenda \& Vinson 1981). However, the correlation of size and age with foraging behaviour is not straightforward, because age of foraging largely depends on size, whereby minor workers are recruited as foragers much earlier than majors, but they are short-lived so that the total amount of time spent foraging by the two types of workers is similar (Tschinkel 2006). Interestingly, in a recent study on the ant Camponotus fellah where individual workers were monitored for 41 days with a tracking system, it has been observed that there is great overlap among groups of workers, with some nurses being older and some foragers being younger than the average workers' age (Mersch et al. 2013). As for metabolism, differential regulation of metabolic genes in workers with different tasks has been found in previous studies on other social insects such as honey bees (Whitfield et al. 2003; Ament et al. 2008; Alaux et al. 2009) and paper wasps (Sumner et al. 2006). In particular, Ament et al. (2011) observed 
that honey bee nurses maintain high levels of lipid and protein metabolism which presumably relate to brood food production, whereas carbohydrate and energy metabolism are held consistently high in foragers to support energy expenses due to flight behaviour. In support of these observations, we found that several GO terms and KEGG pathways related to carbohydrate and energy metabolism varied with foraging/nonforaging behaviours across our two experiments (Table S2 and S4, Supporting Information). For a discussion of genes of interest associated with the metabolism of lipids and carbohydrates, see Appendix S1 (Supporting Information).

Whitfield et al. (2003) reported that honey bee nurses had higher expression of genes associated with neurogenesis, probably due to their role in changing brain structure before the shift to foraging activity, and that nurses and foragers differed for the regulation of genes involved in intracellular signalling in the brain. Consistent with this hypothesis, we found that S. invicta workers with foraging/nonforaging tasks significantly differed in expression of genes associated with larval central nervous system remodelling and signal transduction [see Appendix S1 (Supporting Information) for a discussion of some of these genes].

\section{Effect of social environment on regulation of worker division of labour}

Interestingly, the distinction between the two worker phenotypes was no longer visible at the transcriptional level in queenless colonies, despite the fact that behaviourally and spatially the two phenotypes were still recognizable (see Fig. S1B, Supporting Information). One possible explanation for this apparent contradiction is that queenless workers were not performing the two behaviours reliably, as a result of a loss of specialization and/or spatial organization. Under this scenario, some foraging workers may have spent time inside the nest (therefore, they were assigned to the nonforaging group) and, vice versa, some nonforaging workers may have occupied the outside portion of the nest; hence, they were assigned to the foraging group. Alternatively, after removing the queen (and potentially interrupting the direct effect of primer pheromone on gene regulation in workers), the behavioural and spatial differences between foraging and nonforaging workers may no longer be regulated at the level of gene expression, but rather rely on other factors such as neuropeptides or metabolites that were previously secreted in the hemol$y m p h$. An additional interpretation is that the two phenomena are uncoupled, that is, the differences in gene expression associated with queen removal are not directly involved in the regulation of task behaviours: however, our analysis of gene expression levels in queenright vs. queenless workers independently of the task performed does not support this hypothesis because no significant differences between the two macrogroups were detected. This analysis instead suggests that there might be increased foraging behaviour in queenless workers because their patterns of gene expression were most similar to those found in queenright foragers (see Table S10, Supporting Information). Similar effects are observed in honey bees, where workers exhibit accelerated behavioural maturation (i.e. increase in the number of workers performing foraging) in the absence of the queen or brood (Page et al. 2012). Finally, it is possible that the observed changes in gene expression are not a direct result of the loss of the queen on workers, but rather on the brood, because the brood in queenless colonies is likely reduced and skewed towards an older age distribution. Regardless of the exact mechanism, the results suggest that the sudden loss of the queen can impact the social and spatial organization of a fire ant colony. Additional studies will be necessary to confirm that loss of the queen does indeed result in behavioural changes in workers in fire ant colonies.

Surprisingly, despite this effect on DOL, the presence of the queen had little effect on gene expression patterns in general. Only a handful of genes were differentially expressed between queenright and queenless workers at FDR $<0.1$ [see Appendix S1 (Supporting Information) for a discussion of these genes]. Our results suggest that while queen presence may impact fire ant worker DOL, the effects at the transcriptional level are still quite limited in comparison with the effects of queens on honey bee workers. In honey bees, exposure to queen pheromone triggers changes in expression of thousands of genes in the worker brain (Grozinger et al. 2003). However, in honey bees, there are several profound primer effects of queen pheromone on workers, including inhibition of ovary activation (Hoover et al. 2003) and reduction in juvenile hormone titres (Pankiw et al. 1998), in addition to reduced behavioural maturation. In fire ants, the queen pheromone has significant effects on gynes within a 24$\mathrm{h}$ window, resulting in large-scale changes in gene expression (Wurm et al. 2010) and physiology (wing shedding and ovary activation, Fletcher \& Blum 1981), but no primer effects have been reported in workers. Because caste differences are highly canalized in fire ants, the primer effects of queen pheromone in this system may have been reduced. The presence of a worker caste that is irreversibly sterile prevents the occurrence of mechanisms of queen-worker conflict for the production of males, as observed in other social insects (Bulmer 1981). This fundamental life history trait 
might have selected for a reduction in the direct control of the queen over the workers' physiology through pheromones. Alternatively, it is possible that other social cues, such as brood presence, compensate for the loss of the queen. Brood (in particular fourth-instar larvae) are known to play an important role in regulating the pace of colony activity in fire ants (including queen's egg-laying rate, Tschinkel 1988) and the presence of equal amounts of brood in both colony fragments in Experiment 2 may have masked or buffered the impact of queen loss. Indeed, in honey bees, brood pheromone has similar primer effects as queen pheromone (reviewed in Grozinger accepted). Finally, it is possible that the treatment time (5 days) was not long enough to see differences in workers at the transcriptional level, although changes in gynes are observed within $24 \mathrm{~h}$ (Wurm et al. 2010) and behavioural changes in workers are observed after $48 \mathrm{~h}$ (Klobuchar \& Deslippe 2002) and within 5 days (Vander Meer \& Alonso 2002) after queen removal.

\section{Conclusions}

Our results indicate that there are indeed consistent transcriptional differences associated with DOL in fire ant workers. Interestingly, there was no significant overlap in the sets of genes associated with DOL in fire ant, honey bee and paper wasp workers, although there is some indication that core physiological processes, such as carbohydrate, protein and lipid metabolism, are similarly regulated across these species. We also provide evidence for the first time that social context, namely presence or absence of the queen, can impact worker DOL in fire ants. While the effect of queen presence on gene expression was limited, it nonetheless suggests that the queen may be producing a primer pheromone that impacts worker behaviour and physiology. These findings further confirm the power of a genomic approach for identifying the subtle effects of primer pheromones. In future, it would be of great interest to test whether the addition of a queen extract (which presumably would contain the queen pheromone) is able to 'rescue' expression profiles in queenless workers and make them more similar to queenright workers. Furthermore, it will be necessary to extend this type of approach to encompass the complex social structure of fire ant colonies, where both monogyny and polygyny occur and are determined by two alternative variants of the same genomic element (Wang et al. 2013). In particular, it will be noteworthy to examine how the interaction between social structure and genetic background impacts worker DOL and gene expression in both monogyne and polygyne colonies and in workers of different size and age.

\section{Acknowledgements}

The authors would like to thank Sarah Kocher (Harvard) and Naomi Altman (Penn State) for assistance with the statistical analysis and Mark Brown, Elli Leadbeater, members of the Brown group and the three anonymous reviewers for providing thoughtful comments that helped improving the quality of the manuscript. The authors would also like to thank Alex McMenamin for performing the qRT-PCR studies. Many thanks to Craig Praul and his team at the Penn State Genomics Core Facility for performing all the steps to prepare RNA samples for microarrays and for providing assistance during RNA extraction and data analysis. A special thanks to Eileen Carroll, Chin-Cheng Yang (Scotty) and all the undergraduate research assistants in the Shoemaker Lab that provided extraordinary support, help and advice during field work and laboratory experiments at the USDA-ARS, CMAVE in Gainesville, Florida. This study was funded by US Department of Agriculture AFRI Award 2009-35302-05301 to DS, CMG and John Wang (Academia Sinica), the Swiss NSF and an ERC advanced grant to LK and by the Société Académique Vaudoise to CL.

\section{References}

Alaux C, Le Conte Y, Adams HA et al. (2009) Regulation of brain gene expression in honey bees by brood pheromone. Genes, Brain, and Behavior, 8, 309-319.

Amdam GV, Norberg K, Fondrk MK, Page RE (2004) Reproductive ground plan may mediate colony-level selection effects on individual foraging behavior in honey bees. Proceedings of the National Academy of Sciences of the United States of America, 101, 11350-11355.

Ament SA, Corona M, Pollock HS, Robinson GE (2008) Insulin signaling is involved in the regulation of worker division of labor in honey bee colonies. PNAS, 105, 4226-4231.

Ament SA, Chan QW, Wheeler MM et al. (2011) Mechanisms of stable lipid loss in a social insect. Journal of Experimental Biology, 214, 3808-3821.

Arnold KE, Owens IP, Goldizen AW (2005) Division of labour within cooperatively breeding groups. Behaviour, 142, 1577-1590.

Benjamini Y, Hochberg Y (1995) Controlling the false discovery rate: a practical and powerful approach to multiple testing. Journal of the Royal Statistical Society. Series B (Methodological), 11, 289-300.

Ben-Shahar Y, Robichon A, Sokolowski MB, Robinson GE (2002) Influence of gene action across different time scales on behavior. Science, 296, 741-744.

Blomquist GJ, Bagnères AG (2010) Insect Hydrocarbons: Biology, Biochemistry, and Chemical Ecology. Cambridge University Press, Cambridge, UK.

Bulmer M (1981) Worker-queen conflict in annual social Hymenoptera. Journal of Theoretical Biology, 93, 239-251.

Corona M, Libbrecht R, Wurm Y et al. (2013) Vitellogenin underwent subfunctionalization to acquire caste and behavioral specific expression in the harvester ant Pogonomyrmex barbatus. PLoS genetics, 9, e1003730.

Dreller C, Page RE Jr, Fondrk MK (1999) Regulation of pollen foraging in honeybee colonies: effects of young brood, stored pollen, and empty space. Behavioral Ecology and Sociobiology, 45, 227-233. 
Duarte A, Weissing FJ, Pen I, Keller L (2011) An evolutionary perspective on self-organized division of labor in social insects. In: Annual Review of Ecology, Evolution, and Systematics, vol. 42(eds Futuyma DJ, Shaffer HB \& Simberloff D), pp. 91-110. Annual Reviews, Palo Alto.

Durkheim E, Coser LA (1997) The Division of Labor in Society. Free Press, New York.

Eliyahu D, Ross KG, Haight KL, Keller L, J L (2011) Venom alkaloid and cuticular hydrocarbon profiles are associated with social organization, queen fertility status, and queen genotype in the fire ant Solenopsis invicta. Journal of Chemical Ecology, 37, 1242-1254.

Fletcher DJ, Blum MS (1981) Pheromonal control of dealation and oogenesis in virgin queen fire ants. Science, 212, 73-75.

Gazda SK, Connor RC, Edgar RK, Cox F (2005) A division of labour with role specialization in group-hunting bottlenose dolphins (Tursiops truncatus) off Cedar Key, Florida. Proceedings of the Royal Society B: Biological Sciences, 272, 135-140.

Grozinger CM (accepted) Honey bee pheromones. In: The Hive and the Honey Bee (ed. Graham J), pp. 1-33. Dadant, Indianapolis.

Grozinger CM, Sharabash NM, Whitfield CW, Robinson GE (2003) Pheromone-mediated gene expression in the honey bee brain. Proceedings of the National Academy of Sciences of the United States of America, 100, 14519-14525.

Hoover SE, Keeling CI, Winston ML, Slessor KN (2003) The effect of queen pheromones on worker honey bee ovary development. Naturwissenschaften, 90, 477-480.

Huang DW, Sherman BT, Lempicki RA (2009a) Bioinformatics enrichment tools: paths toward the comprehensive functional analysis of large gene lists. Nucleic acids research, 37, 1-13.

Huang DW, Sherman BT, Lempicki RA (2009b) Systematic and integrative analysis of large gene lists using DAVID bioinformatics resources. Nature Protocols, 4, 44-57.

Johnson BR, Linksvayer TA (2010) Deconstructing the superorganism: social physiology, groundplans, and sociogenomics. The Quarterly Review of Biology, 85, 57-79.

Jouvenaz D, Allen G, Banks W, Wojcik DP (1977) A survey for pathogens of fire ants, Solenopsis spp., in the southeastern United States. The Florida Entomologist, 60, 275-279.

Keeling CI, Slessor KN, Higo HA, Winston ML (2003) New components of the honey bee (Apis mellifera L.) queen retinue pheromone. Proceedings of the National Academy of Sciences, 100, 4486-4491.

Klobuchar EA, Deslippe RJ (2002) A queen pheromone induces workers to kill sexual larvae in colonies of the red imported fire ant (Solenopsis invicta). Naturwissenschaften, 89, 302-304.

Le Conte Y, Bécard J-M, Costagliola G et al. (2006) Larval salivary glands are a source of primer and releaser pheromone in honey bee (Apis mellifera L.). Naturwissenschaften, 93, 237-241.

Libbrecht R, Oxley PR, Kronauer DJ, Keller L (2013) Ant genomics sheds light on the molecular regulation of social organization. Genome biology, 14, 212.

Lucas C, Sokolowski MB (2009) Molecular basis for changes in behavioral state in ant social behaviors. Proceedings of the National Academy of Sciences, 106, 6351-6356.

Lucas C, Hughson BN, Sokolowski MB (2010) Job switching in ants: role of a kinase. Communicative \& Integrative Biology, 3, 6-8.

Manfredini F, Riba-Grognuz O, Wurm Y et al. (2013) Sociogenomics of cooperation and conflict during colony founding in the fire ant Solenopsis invicta. PLoS genetics, 9, e1003633.
Manser MB (1999) Response of foraging group members to sentinel calls in suricates, Suricata suricatta. Proceedings of the Royal Society of London. Series B: Biological Sciences, 266, 1013-1019.

Marygold SJ, Leyland PC, Seal RL et al. (2013) FlyBase: improvements to the bibliography. Nucleic Acids Research, 41, D751-DD757.

Mersch DP, Crespi A, Keller L (2013) Tracking individuals shows spatial fidelity is a key regulator of ant social organization. Science, 340, 1090-1093.

Mirenda JT, Vinson SB (1981) Division of labour and specification of castes in the red imported fire ant Solenopsis invicta Buren. Animal Behaviour, 29, 410-420.

Oliveros J.C. (2007) VENNY. An interactive tool for comparing lists with Venn Diagrams. http://bioinfogp.cnb.csic.es/ tools/venny/index.html.

Page J, Robert E, Rueppell O, Amdam GV (2012) Genetics of reproduction and regulation of honeybee (Apis mellifera L.) social behavior. Annual Review of Genetics, 46, 97-119.

Pankiw T, Page RE (2001) Genotype and colony environment affect honeybee (Apis mellifera L.) development and foraging behavior. Behavioral Ecology and Sociobiology, 51, 87-94.

Pankiw T, Huang Z, Winston M, Robinson G (1998) Queen mandibular gland pheromone influences worker honey bee (Apis mellifera L.) foraging ontogeny and juvenile hormone titers. Journal of insect physiology, 44, 685-692.

Robinson GE (1992) Regulation of division of labor in insect societies. Annual Review of Entomology, 37, 637-665.

Robinson GE, Grozinger CM, Whitfield CW (2005) Sociogenomics: social life in molecular terms. Nature Reviews Genetics, 6, 257-270.

Slessor KN, Winston ML, Le Conte Y (2005) Pheromone communication in the honeybee (Apis mellifera L.). Journal of Chemical Ecology, 31, 2731-2745.

Smith CR, Toth AL, Suarez AV, Robinson GE (2008) Genetic and genomic analyses of the division of labour in insect societies. Nature Reviews Genetics, 9, 735-748.

Sturn A, Quackenbush J, Trajanoski Z (2002) Genesis: cluster analysis of microarray data. Bioinformatics, 18, 207-208.

Sumner S, Pereboom JJ, Jordan WC (2006) Differential gene expression and phenotypic plasticity in behavioural castes of the primitively eusocial wasp, Polistes canadensis. Proceedings of the Royal Society B: Biological Sciences, 273, 19-26.

Team RDC (2009) R: a Language and Environment for Statistical Computing. R Foundation for Statistical Computing, Vienna, Austria.

Toth AL, Varala K, Newman TC et al. (2007) Wasp gene expression supports an evolutionary link between maternal behavior and eusociality. Science, 318, 441-444.

Toth AL, Varala K, Henshaw MT et al. (2010) Brain transcriptomic analysis in paper wasps identifies genes associated with behaviour across social insect lineages. Proceedings of the Royal Society B: Biological Sciences, 277, 2139-2148.

Tschinkel WR (1988) Social control of egg-laying rate in queens of the fire ant, Solenopsis invicta*. Physiological Entomology, 13, 327-350.

Tschinkel WR (2006) The Fire Ants. The Belknap Press of Harvard University Press, Cambridge, Massachusetts.

Vander Meer RK, Alonso LE (2002) Queen primer pheromone affects conspecific fire ant (Solenopsis invicta) aggression. Behavioral Ecology and Sociobiology, 51, 122-130. 
Vander Meer R, Glancey B, Lofgren C et al. (1980) The poison sac of red imported fire ant queens: source of a pheromone attractant. Annals of the Entomological Society of America, 73, 609-612.

Vander Meer RK, Breed MD, Espelie KE, Winston ML (1998) Pheromone Communication in Social Insects. Westview Press, Colorado.

Vargo EL (1992) Mutual pheromonal inhibition among queens in polygyne colonies of the fire ant Solenopsis invicta. Behavioral Ecology and Sociobiology, 31, 205-210.

Vargo EL (1998) Primer pheromones in ants. In: Pheromone Communication in Social Insects: Ants, Wasps, Bees, and Termites, (eds Vander Meer R, Breed MD, Espelie KE, Winston M), pp. 293-313. Westview Press, Boulder, Colorado.

Vargo EL, Fletcher DJ (1986a) Evidence of pheromonal queen control over the production of male and female sexuals in the fire ant, Solenopsis invicta. Journal of Comparative Physiology $A, \mathbf{1 5 9}, 741-749$.

Vargo EL, Fletcher DJ (1986b) Queen number and the production of sexuals in the fire ant, Solenopsis invicta (Hymenoptera: Formicidae). Behavioral Ecology and Sociobiology, 19, 41-47.

Vargo EL, Fletcher DJ (1987) Effect of queen number on the production of sexuals in natural populations of the fire ant, Solenopsis invicta. Physiological Entomology, 12, 109-116.

Wang J, Wurm Y, Nipitwattanaphon M et al. (2013) A Y-like social chromosome causes alternative colony organization in fire ants. Nature, 493, 664-668.

Whitfield CW, Cziko A-M, Robinson GE (2003) Gene expression profiles in the brain predict behavior in individual honey bees. Science, 302, 296-299.

Wilson EO (1971) The insect societies. Harvard University Press, Cambridge, Massachusetts.

Wurm Y, Wang J, Keller L (2010) Changes in reproductive roles are associated with changes in gene expression in fire ant queens. Molecular Ecology, 19, 1200-1211.

Wurm Y, Wang J, Riba-Grognuz O et al. (2011) The genome of the fire ant Solenopsis invicta. Proceedings of the National Academy of Sciences of the United States of America, 108, 5679-5684.

Wyatt TD (2003) Pheromones and Animal Behaviour: Communication by Smell and Taste. Cambridge University Press, Cambridge, UK.

F.M., C.L., L.K., D.S. and C.M.G. designed research. F.M., C.L., M.N. and D.S. performed research. F.M. and C.M.G. analysed the data. F.M. and C.M.G. wrote the manuscript. C.L., L.K. and D.S. provided comments on the manuscript.

\section{Data accessibility}

The array data were deposited on the ArrayExpress database (www.ebi.ac.uk/arrayexpress) according to MIAME standards (accession numbers: E-MTAB-2094 for Experiment 1 and E-MTAB-2108 for Experiment 2). Gene expression data are provided as Appendix S1 (Supporting Information) with this article.

\section{Supporting information}

Additional supporting information may be found in the online version of this article.

Fig. S1 Experimental set-up for Experiment 2. A) Four mother colonies were split into 2 colony fragments each. All colony fragments were provided with a nesting chamber, a foraging area with a cricket, water and sugar water, and equal amounts of workers and brood. B) Queenless colony fragment. The queen is no longer inside the nesting chamber, but some workers continue to perform in-nest tasks. $\mathrm{QR}=$ queenright; $\mathrm{QL}=$ queenless; in = nonforaging workers; out = foraging workers.

Fig. S2 Microarray hybridization scheme for Experiment 1. For each group of workers, 6 pools were hybridized in a loop design: 3 pools were labelled with the Cy3 dye and other 3 with the Cy5 dye. We used 6 arrays of a 12-plex array slide with 135000 probe capacity designed by Roche NimbleGen, Inc. (Madison WI)

Fig. S3 Microarray hybridization scheme for Experiment 2. For each group of workers, 6 pools were hybridized in a loop design: 3 pools were labelled with the $\mathrm{Cy} 3$ dye and other 3 with the Cy5 dye. We used a whole 12-plex array slide with 135000 probe capacity designed by Roche NimbleGen, Inc. (Madison WI).

Fig. S4 Validation of analysis of gene expression with $\mathrm{R}$ in samples from Experiment 1. Analysis with $R$ produced almost twice the number of transcripts differentially expressed between foraging and nonforaging workers at FDR $<0.05$ than analysis with SAS (1387 vs. 771, respectively). However, a comparative analysis between the two set of transcripts revealed large overlap confirming that the choice of either analysis would not affect the biological significance of the results.

Fig. S5 Comparative analysis of sets of transcripts differentially expressed at FDR $<0.05$ in both Experiments 1 and 2. A pool of 89 transcripts were shared (more than expected by chance, hypergeometric test: representation factor: 8.4, $P<7.16 \mathrm{e}-55)$, but these did not produce any significantly enriched GO terms. GO terms indicated in the figure refer to the two sets of transcripts analysed separately.

Fig. S6 Quantitative real-time PCR validation of expression levels of genes of interest. A) Expression levels of the following genes associated with GO terms of interest were analysed using quantitative real-time PCR (see Table S10 for detailed information about these genes and the primers we used): For (food-related behaviour and polyethism), Hym (antibacterial response), $m f$ (muscle development), mhc (locomotion), oat (neurogenesis), spirit (innate immune response) and syt1 (neurotransmitter secretion). Mean expression levels in QLin and QRout were normalized to levels of expression in QRin workers. We used 9 pools for QLin, 8 for QRin and 5 for QRout, each pool being composed by 10 workers from the same colonies used for Experiment 2 (but different individuals). Statistical analysis was performed with nonparametric Wilcoxon comparisons for each pair of treatments: $*=P<0.05$; $* *=$ $P<0.01$. \#For a better visualization of the results, the bar associated with the gene Hym is not represented in full length in 
QRout: average relative expression for this gene was 3.95 with S.E. \pm 0.94 ; $\mathrm{QRin}=$ queenright nonforaging workers; $\mathrm{QLin}=$ queenless nonforaging workers; QRout = queenright foraging workers. B) $\log _{2}$-transformed and normalized expression values for the same genes as above after microarray analysis.

Table S1 Experiment 1: differentially expressed transcripts between foraging vs. nonforaging workers at FDR $<0.05$.

Table S2 Experiment 1: significantly enriched GO terms and KEGG pathways (functional annotation chart, $P<0.05$ ).

Table S3 Experiment 2: differentially expressed transcripts between queenright nonforaging (QRin) and foraging (QRout) workers at FDR $<0.05$.

Table S4 Experiment 2: significantly enriched GO terms and KEGG pathways (functional annotation chart, $P<0.05$ ).

Table S5 Experiment 2: differentially expressed transcripts between queenright and queenless workers at FDR $<0.1$. The list includes transcripts that were differentially expressed either in the QRin-QLin or in the QRout-QLout comparisons.

Table S6 Differentially expressed transcripts that were shared at FDR $<0.05$ between Experiment 1 and Experiment 2 .
Table S7 Comparisons of significantly enriched GO terms from Experiment 1 and Experiment $2(P<0.05)$.

Table S8 Overlap analysis between 17960 transcripts upregulated in queenless workers and transcripts that were upregulated in foraging and nonforaging workers.

Table S9 Comparative analysis of the 735 transcripts provided with FlyBase annotations that were differentially expressed in foraging vs. nonforaging workers in Experiment 1 and in other previous transcriptome studies.

Table S10 Quantitative real-time PCR validation of expression levels of genes of interest in workers from Experiment 2: gene lists and primers' sequences.

Appendix S1 Supporting information for online publication including further details (with relevant references) on the following aspects: insect collection, rearing and sampling; sample preparation for molecular analyses; protocols for microarray analysis; validation of differential expression of candidate genes using quantitative real-time PCR; comparative studies across species; discussion of genes of interest. 